Philosophie ANTIQUE

\section{Philosophie antique}

Problèmes, Renaissances, Usages

$19 \mid 2019$

L'épicurisme antique

\title{
Sexe, amour et politique chez Lucrèce
}

\section{Pierre-Marie Morel}

\section{(2) OpenEdition}

\section{Journals}

Édition électronique

URL : https://journals.openedition.org/philosant/3093

DOI : 10.4000/philosant.3093

ISSN : 2648-2789

\section{Éditeur}

Éditions Vrin

\section{Édition imprimée}

Date de publication : 31 octobre 2019

Pagination : $57-84$

ISBN : 978-2-7574-2534-3

ISSN : 1634-4561

\section{Référence électronique}

Pierre-Marie Morel, « Sexe, amour et politique chez Lucrèce », Philosophie antique [En ligne], 19 | 2019, mis en ligne le 31 octobre 2020, consulté le 02 décembre 2022. URL : http://journals.openedition.org/ philosant/3093 ; DOI : https://doi.org/10.4000/philosant.3093

\section{cc) (1) 8}

Creative Commons - Attribution - Pas d'Utilisation Commerciale - Pas de Modification 4.0 International - CC BY-NC-ND 4.0

https://creativecommons.org/licenses/by-nc-nd/4.0/ 


\section{SEXE, AMOUR ET POLITIQUE CHEZ LUCRÈCE Pierre-Marie MOREL \\ Université Paris I-Panthéon Sorbonne}

RÉSUMÉ. Cet article cherche à montrer qu'amour et politique sont étroitement liés dans le De natura rerum de Lucrèce. D'une part, l'amour-passion, au Livre IV, se révèle aussi vain que le désir du pouvoir politique; d'autre part, le livre $\mathrm{V}$ oppose implicitement le désir sexuel des premiers êtres humains au «bien commun » qui gouverne les organisations sociales. En outre, la description, au livre IV, d'une sexualité exempte de passion, ou amour libre, caractérisée par un désir mutuel et un plaisir partagé, fait office de contre-modèle ou de paradigme alternatif face à l'aliénation et à la violence politiques et sociales. En d'autres termes, l'objet de cet article est de montrer qu'amour, sexe et politique font l'objet chez Lucrèce d'une même approche, où l'on peut voir à la fois une composante importante de sa thérapie de l'âme et une profonde défiance à l'égard de la vie sociale et des activités politiques.

Summary. The aim of this article is to show that love and politics are closely connected in Lucretius' poem De rerum natura. On the one hand, passionate love (Book IV) is similar to the vain desire for political power; on the other hand, sexual desire of primitive human beings (in Book $V$ ) is implicitely opposed to the 'common good' which governs human societies and cities. Moreover, the description, in Book IV, of non passionate sexuality, or free love, which is characterized by mutual desire and shared pleasure, is a sort of counter-model, or an alternative paradigm, in opposition to political and social alienation and violence. In other words, it is argued, Lucretius' global attitude to love, sex and politics is an important feature of the therapy of the soul. Accordingly, it reveals a deep suspicion in face of social life and political activities.

Philosophie antique, $\mathrm{n}^{\circ} 19$ (2019), 57-84 

L'opposition entre la vie politique et l'idéal de relations humaines plus sûres pour la tranquillité de l'âme est l'un des aspects les mieux connus de la conception épicurienne de la vie bonne ${ }^{1}$. Face aux dangers venus de la cité, les épicuriens invitent à une sociabilité restreinte, au sein d'une communauté d'amitié, ainsi que le proclame la fameuse exhortation d'Épicure à « vivre caché $»^{2}$. Il est vrai que les épicuriens n'ont pas rejeté toute forme d'intérêt pour la politique, contrairement à ce que laissent croire les critiques qui leur ont été adressées dans l'Antiquité par Cicéron ou Plutarque. Les tendances actuelles de l'interprétation invitent d'ailleurs à nuancer le clivage entre vie publique et retrait vers la sphère privée ${ }^{3}$. Quoi qu'il en soit, de l'interprétation que l'on donne à cette opposition dépendent en grande partie notre compréhension de la politique des épicuriens de l'Antiquité et, plus généralement, celle de leur conception de la sociabilité et des rapports humains.

Ce problème d'interprétation, dans ses grandes lignes, concerne bien entendu les écrits d'Épicure, mais également le De rerum natura (DRN) de Lucrèce, où la question plus générale du rapport à autrui se pose constamment. Chez ce dernier, les différentes formes de relations humaines ne sont pas toutes également représentées. Ainsi, l'amitié, en dehors du fait que Lucrèce dédie son poème à un ami, Memmius ${ }^{4}$, est rarement évoquée de

1. J'entends provisoirement par « vie politique » aussi bien la vie de simple citoyen que les activités liées à l'exercice du pouvoir. Des versions préparatoires de ce texte ont été présentées dans le Séminaire de Philosophie Hellénistique et Romaine, à Paris, en 2007, et lors du colloque Lucretius poet and philosopher: six hundred years from his rediscovery, à Alghero (Italie) en 2017. Je remercie les amis et collègues qui ont bien voulu me faire part de leurs observations en ces occasions, et en tout premier lieu Alain Gigandet.

2. Fragment Usener (désormais Us.) 551. Voir Morel 2007, sur la notion de communauté « restreinte », pour exprimer l'idée que la cité n'est pas le cadre naturel de la sociabilité humaine souhaitable, celle-ci se réalisant dans une sphère plus étroite, en l'occurrence celle de l'amitié.

3. Voir par exemple, dans la littérature récente, Roskam 2007, Brown 2009, Fish 2011, Morel 2000, Morel 2017.

4. Lucrèce, DRN I, 26. 
manière directe. La vie dans les regroupements primitifs puis dans les cités l'est un peu plus, mais la politique proprement dite n'est abordée, le plus souvent, que de manière allusive. En revanche, la vie amoureuse - entendue pour l'instant au sens large, incluant à la fois les relations strictement physiques et les relations fondées sur un sentiment amoureux - est l'objet d'une attention explicite et forte.

En première approche, les observations éparses de Lucrèce sur les différentes formes de relations humaines ne permettent pas de reconstituer une théorie générale du rapport à autrui. À l'examen, cependant, ces différents types de situations ne sont pas sans liens. Ils esquissent ce que le poète attend ou redoute de l'altérité humaine en général. Il n'y a d'ailleurs pas lieu de s'en étonner, si l'on admet, d'une part, que les différents types de relations humaines sont étroitement reliés aux passions qu' ils suscitent et, d'autre part, que celles-ci ne sont pas sans rapports les unes avec les autres. Je voudrais précisément défendre ici l'idée que, dans le poème de Lucrèce, la typologie des relations amoureuses est en rapport direct avec les problèmes que pose la vie en société. La question de l'amour, en effet, donne des indications significatives sur ce que devrait être une relation positive à autrui, mais également sur l'attitude qu' il convient d'adopter face aux relations de pouvoir, en particulier face au pouvoir politique. En d'autres termes, la problématique amoureuse, dans le poème de Lucrèce, est non seulement traitée pour elle-même, mais également pour ce qu'elle révèle des passions dans leur ensemble, en particulier celles qui animent la « vie politique » sous ses différents aspects.

Avant d'entrer dans l'analyse proprement dite des textes de Lucrèce, rappelons les termes généraux du débat d'interprétation. L'ensemble que constituent les passages sur l'amour, envisagé sous ses différentes formes - la passion amoureuse, la sexualité sans attachement (primitive ou non), et la relation conjugale -, atteste par son ampleur et son caractère explicite l'intérêt que Lucrèce porte à la question. On ne peut en dire autant, je l'ai dit, de la politique. Il est vrai que l'on peut déceler, comme plusieurs spécialistes l'ont fait, des messages politiques dans le $D R N$, notamment une critique implicite de la politique romaine ${ }^{5}$. Cependant, ces messages supposés restent souvent allusifs. De plus, la question de la justice politique ne fait pas l'objet d'une analyse systématique, ni même simplement continue, mais plutôt de métaphores et de remarques formulées comme en passant. De fait, le $D R N$ ne donne pas d'indications nettes permettant de dire si Lucrèce a pris position sur la nature du contrat qui devrait réunir les membres d'une communauté politique juste. C'est une différence notable par rapport aux maximes

5. Pour une synthèse pondérée de la question, voir Schiesaro 2007. Voir également Fish 2011 qui, pour sa part, repère dans le $D R N$ plusieurs indices de l'acceptation d'une politique légitime, parmi lesquels les activités politiques de Memmius. 
d'Épicure consacrées aux règles de droit $^{6}$, maximes qui mettent en avant le caractère contractuel des relations à l'intérieur de la communauté politique. Nous y reviendrons. Dans le $D R N$, les critiques de la vie politique et de l'abus de pouvoir sont claires (comme dans le prélude du chant II, au chant III ou au chant $\mathrm{V}$ ), mais cela nous dit très peu des conditions concrètes de réalisation d'une cité juste.

Significativement, D. Fowler, dans un article fondamental pour l'analyse du dossier, argumente dans un sens minimaliste, estimant que le message du poème concerne avant tout le salut individuel. On retiendra en particulier l'expression par laquelle il qualifie la position de Lucrèce à l'égard de la politique et des sociétés humaines : « a realistically sceptical view of social institutions $»^{7}$. G. Roskam, dans son étude de référence sur le « vivre caché » épicurien, est allé plus loin encore que Fowler en minimisant fortement la place d'une éventuelle politique positive dans le poème de Lucrèce, et en la réduisant en substance à la recommandation du chant $\mathrm{V}:$ mieux vaut « obéir paisiblement » (parere quietum) plutôt que vouloir dominer le monde et diriger des royaumes ${ }^{8}$.

L'hypothèse de travail que j'ai proposée plus haut consiste à partir, non pas des rares indications ou observations de Lucrèce sur la politique ellemême, mais du rapport à autrui tel que le révèlent, en particulier, les relations amoureuses. Pour autant que je puisse en juger, le rapport entre l'amour et la politique est rarement traité de manière approfondie par les commentateurs. Il est notamment révélateur que $\mathrm{R}$. D. Brown se montre très discret sur la question politique dans son commentaire, pourtant très savant et très complet, de la section du chant IV du $D R N$ sur la sexualité et l'amour passionnel ${ }^{9}$. De fait, la relation amoureuse n'intervient pas dans la construction traditionnelle de la position épicurienne concernant la politique et les cités humaines, qu'il s'agisse des Maximes Capitales XXXI-XXXVIII d'Épicure ou du long texte de Porphyre témoignant de la généalogie, par l'épicurien Hermarque, des sociétés humaines ${ }^{10}$.

Je voudrais cependant mettre en évidence à ce propos deux points qui concernent, non seulement la politique et la passion amoureuse, mais encore la philosophie lucrétienne des passions ou émotions dans son ensemble. En

6. Voir Épicure, Maximes Capitales (désormais $M C$ ) XXXI-XXXVIII.

7. Fowler 1989, p. 147.

8. DRNV, 1129-1130. Roskam 2007, p. 97 et, sur l'ensemble de la question politique chez Lucrèce, p. 86-99.

9. Voir Brown 1987. Baier 2010, dans son étude sur l'irrationnel dans le $D R N$, ne consacre à la question politique qu'un paragraphe (p. 102-103), dans lequel il estime que l'amour est essentiellement pour Lucrèce une question individuelle, et que la folie amoureuse ne met pas réellement en péril les institutions sociales. Je reviendrai plus bas sur ce dernier point, qui me paraît discutable.

10. Porphyre, Abst. I, 7-12. 
premier lieu, si l'on prend en compte, non seulement le chant IV, mais également d'autres passages sur les relations amoureuses, en particulier le chant $\mathrm{V}$, on constate que ces deux thèmes se croisent effectivement dans le texte de Lucrèce. On perçoit en effet des analogies significatives entre l'amour et les situations politiques, par exemple entre le désir amoureux passionnel et l'appétit de pouvoir. M. Nussbaum a utilement attiré l'attention sur ce qui, selon elle, relève de l'anthropologie politique dans l'évocation lucrétienne de l'amour conjugal ${ }^{11}$. Elle y voit le signe d'une construction progressive des relations sociales, à partir du couple et de la structure familiale, dont Vénus accompagnerait les différentes étapes. Cette évolution est bien marquée, en effet, dans le chant $\mathrm{V}$. Toutefois, elle ne permet pas de voir dans l'accouplement un véritable principe du lien social. Je ne vois pas en tout cas dans le texte de Lucrèce, à la différence notamment de ce que l'on trouve chez Aristote, de claire continuité entre la constitution primitive du couple et celle de la cité. Contre la lecture continuiste défendue par M. Nussbaum, j'insisterai à l'inverse, non pas sur la dimension positive de la première union, mais sur la critique de la politique qu'inspire la réflexion lucrétienne sur l'amour. En un mot, j'estime que l'amour a chez Lucrèce une dimension politique, mais que cette dimension est essentiellement critique et négative : l'amour passionnel présente les mêmes symptômes pathologiques que les désirs politiques, tandis que l'amour libre et l'amour primitif sont en opposition radicale avec le mode de vie « politique » au sens large.

Après avoir brièvement rappelé l'arrière-plan doctrinal concernant la question des relations amoureuses dans l'épicurisme ${ }^{12}, \mathrm{j}^{\prime}$ examinerai les textes témoignant du rapport entre amour et politique, d'abord dans le chant $\mathrm{V}$, puis dans le chant IV.

\section{Sur l'épicurisme et l'amour}

D'Épicure à Lucrèce, la passion amoureuse est présentée, explicitement ou implicitement, comme une émotion en quelque sorte « asociale », et cela à deux niveaux. À un premier niveau, on constate que l'amour en général ne contribue pas à l'harmonie collective, dans la mesure où il n'œuvre pas à la réalisation du juste, c'est-à-dire à ce qui est utile à une communauté donnée. Je me réfere ici à la définition épicurienne du juste, le dikaion, telle qu'elle apparaît dans les maximes d'Épicure déjà mentionnées. On notera que, chez Hermarque, la généalogie de l'humanité fait peu de place à l'accouplement, qui ne paraît pas jouer un rôle décisif dans la constitution des sociétés. La relation amoureuse, de ce point de vue, est « asociale » au sens où elle est

11. Voir Nussbaum 1994 et plus bas, p. 73-74; 79-80.

12. Voir en particulier la synthèse de Brown 1987, p. 101-122. 
socialement neutre. Toutefois, à un second niveau, le sentiment amoureux peut avoir pour effet d'éloigner les individus les uns des autres et de briser les liens relevant de la philia. La passion amoureuse conduit à un désordre non seulement psychique, mais aussi économique, éthique et politique. L'amant dilapide ses biens et néglige ses devoirs, ses officia ${ }^{13}$.

Sous ce double point de vue, l'eros épicurien se distingue nettement de l'eros stoïcien. Ce dernier prend place, à partir de la conformation des organes du corps en vue de la reproduction ${ }^{14}$ et de la pulsion sexuelle, puis par la médiation du mariage, dans la chaîne qui relie - au moins idéalement les tendances naturelles à l'harmonie politique et cosmique ${ }^{15}$. On ne trouve pas, dans les textes épicuriens actuellement disponibles, quelque chose qui ressemblerait à la 'city of love', la cité de l'eros, qu'évoque M. Schofield dans son analyse de la République de Zénon ${ }^{16}$. Il y aurait peut-être un point de rapprochement entre stoïciens et épicuriens dans l'idée que les relations sexuelles non exclusives évitent les complications liées à la jalousie que suscite l'adultère, ainsi que l'indique à propos des stoïciens un témoignage de Diogène Laërce ${ }^{17}$. On retrouverait là, en effet, quelque chose qui ressemble à la dépersonnalisation de la relation amoureuse prônée par Lucrèce lorsqu'il évoque les avantages de la Vénus volage, sur lesquels nous reviendrons. Toutefois, dans la sociabilité stoïcienne telle que l'évoque le passage de Diogène Laërce sur l'absence de jalousie, l'affection égale des hommes pour tous les enfants issus de ces relations, « comme si nous étions le père », joue manifestement un rôle positif en vue du renforcement du lien social ${ }^{18}$. Nous verrons que, chez Lucrèce, la sexualité sans attachement n'a pas de fonction de cet ordre. On peut également supposer que, chez les stoïciens, la relation sexuelle, dans le contexte de la relation pédagogique et en compagnie du sage, conduit à l'amitié, pour autant que le plaisir sexuel lui-même soit considéré comme un indifférent ${ }^{19}$. Cette manière d'assumer sans passion les relations sexuelles présente à première vue une certaine similitude avec la position que défend Lucrèce. Toutefois, chez ce dernier, l'amour libre reste sans suite sur le plan relationnel. La relation amoureuse, loin d'œuvrer à la cohésion sociale et

13. DRN IV, 1121-1132.

14. Voir par exemple Cicéron, Fin. III, 62.

15. On consultera désormais sur ce point Laurand 2014.

16. Schofield 1991, p. 22-56.

17. Diogène Laërce, VII, 131.

18. Les textes du stoïcien Hiéroclès présentent une approche systématique du rapport qui relie l'attachement entre les générations aux devoirs à l'égard de la patrie et des autres hommes, jusqu’à l'humanité tout entière. Voir sur ce point Veillard 2016, p. 106 qui précise : « le transfert des liens d'attachement et la hiérarchisation subséquente des devoirs s'expliquent par le principe d'engendrement, plus précisément par la transitivité de l'engendrement... »

19. Voir Diogène Laërce, VII, 130 ; Laurand 2005, p. 70-71 ; Laurand 2014, p. 230, 254-255, 259. 
politique, disjoint nettement eros et philia, que ce soit par opposition au stoïcisme ou par contraste avec l'érotique platonicienne. De fait, il est très clair qu'à la différence de l'eros platonicien, tel qu'il apparaît par exemple dans le Banquet, le traitement de l'amour dans le De rerum natura a pour double caractéristique d'être indépendant de la question de l'aspiration à la philosophie et de disjoindre l'amour physique de sa dimension affective et relationnelle. Je cite sur ce point l'appréciation très claire d'Alain Gigandet :

L'amour passionné est destructeur du lien social et politique, l'amant néglige ses amis, ses devoirs civiques, etc. Tout cela est pris dans la problématique éthique proprement épicurienne de l'utile et du bonheur : faire son malheur en pensant poursuivre son bonheur, c'est la traduction pratique de l'illusion épistémologique. Mais l'essentiel semble être le principe d'où procède le thème diatribique, la dissociation radicale d'eros et de philia, que Platon prétendait articuler ${ }^{20}$.

On évitera en tout cas, dès la première approche, la double caricature qui opposerait, à un Portique asexué, un Jardin de jouissances et de débauches ${ }^{21}$.

La vision commune d'un épicurisme préconisant une sexualité sans restriction est assez tenace, mais les textes que nous allons aborder maintenant montrent qu'il n'est pas difficile de l'écarter. L'attitude épicurienne à l'égard de l'amour en général laisse en effet paraître, dès l'origine, une certaine ambivalence.

D’un côté, on pourra évoquer certains aspects de la vie du Jardin au temps d'Épicure, comme les liens des uns et des autres avec différentes prostituées intégrées à la communauté, et dont les noms figurent dans la biographie d'Épicure chez Diogène Laërce ${ }^{22}$. De plus, les propos d'Épicure lui-même confortent parfois l'image d'un hédonisme sans réserve, comme cette déclaration extraite de son traité Sur la fin:

En tout cas, personnellement, je ne parviens pas à concevoir ce que peut être le bien, si j'élimine les plaisirs que procurent les saveurs, si j'élimine ceux du sexe, si j'élimine ceux que procurent les sons, et si j'élimine les mouvements agréables que suscite la vue de la beauté des formes ${ }^{23}$.

D’un autre côté, de telles proclamations sont fortement pondérées par un certain nombre de textes dont la tonalité est beaucoup plus austère ${ }^{24}$.

20. Gigandet 1999, p. 78 ; voir également Giovacchini 2014.

21. Sur les caricatures dont l'épicurisme a fait l'objet à partir de l'Antiquité, on pourra se reporter à Gordon 2012.

22. Diogène Laërce, $X, 7$. Notons toutefois qu'Épicure adresse des lettres à certaines d'entre elles (Diogène Laërce, $\mathrm{X}, 6$ ), comme à n'importe quel interlocuteur philosophique.

23. Épicure cité par Athénée, Deipnosophistes, XII, 546 e (Us. 67). Voir aussi Diogène Laërce, X, 6 ; Cicéron, Tusculanes, III, 41.

24. L'ambiguïté sur la valeur de l'eros est manifeste si l'on prend en compte les textes rassemblés par Usener sous son numéro 483, où l'amour, eros, est défini comme « désir intense des aphrodisia » et paraît à ce titre condamné par Épicure, par opposition à l'amitié. Voir 
On trouve en effet dans le corpus épicurien tout un ensemble de formules restrictives, voire prohibitives, à l'égard des relations amoureuses en général, au nom de la tranquillité psychique. Il est donc inutile de rappeler ici tout ce que les condamnations morales de l'épicurisme peuvent avoir d'excessif. L'éthique épicurienne, on le sait, prône en réalité un calcul prudent des plaisirs. Tout plaisir n'a pas à être choisi, comme le dit notamment Épicure dans la Lettre à Ménécée, aux paragraphes 131-132:

Quand donc nous disons que le plaisir constitue la fin, nous ne parlons pas des plaisirs des débauchés ni de ceux qui consistent dans les jouissances, comme le croient certains qui ignorent de quoi nous parlons, sont en désaccord avec nos propos ou les prennent dans un sens qu'ils n'ont pas, mais du fait de ne pas souffrir, pour le corps, [132] et de ne pas être troublé, pour l'âme. En effet, ce ne sont ni l'incessante succession des beuveries et des parties de plaisir, ni les jouissances que l'on trouve auprès des garçons et des femmes, ni celles des poissons et de toutes les autres choses que présente une table abondante, qui font que la vie est agréable : c'est un raisonnement sobre, qui recherche la connaissance exacte des raisons de tout choix et de tout refus et qui rejette les opinions à partir desquelles la plus grande confusion s'empare des âmes ${ }^{25}$.

Pour Épicure, le désir sexuel en lui-même fait partie des désirs naturels non nécessaires, c'est-à-dire ceux dont la non-satisfaction n'est pas douloureuse ${ }^{26}$. Il semble être même allé plus loin dans cette restriction, si l'on se réfère au fragment 456 des Epicurea d'Usener : « le désir sexuel est naturel, mais il

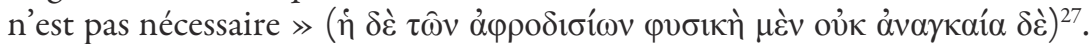
Le texte précise, de manière assez opaque, que les désirs d'un certain type de

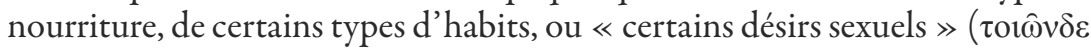
$\left.\grave{\alpha} \varphi \rho \delta_{1 \sigma} i^{\omega} \omega\right)$ ne sont ni naturels ni nécessaires. En d'autres termes, tous ces désirs sont dans leur principe justifiables, même s’ils ne sont pas « néces-

Us. 483 (Hermias, In Plat. Phaedr., 76 ; Alexandre d'Aphrodise, In Ar. Top., 75 ; Scholies à Denys de Thrace, 667, 13 ; Cicéron, Tusc. IV, 33, 70).

25. Traduction Morel 2011.

26. Voir Cicéron, Tusc. V, 94 : « d'une façon générale, les plaisirs de cette catégorie sont désirables, à condition qu'ils ne puissent faire du tort, mais ils ne sont jamais utiles » (Omninoque genus hoc voluptatum optabile esse, si non obsit, prodesse numquam trad. Humbert 1931). Que le désir sexuel soit naturel sans être nécessaire peut aisément se concevoir, si l'on admet qu'il ne vise pas un plaisir stable ou catastématique, mais un plaisir cinétique, c'est-à-dire une satisfaction par variation ou compensation. Voir en ce sens Landolfi 2013, p. 14, ouvrage qui offre par ailleurs une synthèse récente, précise et utile sur l'ensemble de ce dossier. Purinton 1993 défend toutefois l'idée que le plaisir sexuel, comme les plaisirs cinétiques en général (par opposition aux états stables ou catastématiques), comme la joie ( $\chi \alpha \rho \alpha ́)$ ou boire quand on a soif, sont désirables pour le sage. Le fragment Us. 67, on l'a vu, va dans ce sens. Cette position est justifiable si l'on admet, comme Purinton, que c'est la possession des plaisirs stables qui nous permet de jouir comme il convient des plaisirs en mouvement.

27. Us. 456 ; Scholies in Ar. Eth. Nic., III, 13, 1118 b8. 
saires », mais certains d'entre eux, en se particularisant, perdent toute justification. S'agit-il simplement de condamner l'adultère ou certaines pratiques sexuelles ? On pourrait le penser, à en juger par la recommandation suivante, malheureusement assez imprécise : « le sage n'aura pas de relations sexuelles avec une femme dans des conditions que les lois interdisent, comme le dit Diogène <de Tarse> dans son Résumé des Doctrines morales d'Épicure ${ }^{28}$. » D'ailleurs, dans cet interdit, les dommages évoqués ne sont pas uniquement psychologiques : ils sont sans doute également sociaux, comme le suggère la mention de la loi.

On peut en tout cas faire l'hypothèse qu'Épicure avait esquissé avant Lucrèce une distinction entre, d'une part, les satisfactions procurées par la sexualité physique, en elle-même non nécessaire mais naturelle, et d'autre part les sentiments passionnels et les excès dans la pratique des aphrodisia.

Dans certains textes, toutefois, les restrictions s'étendent au-delà du cas de la passion passagère et des excès, dont les inconvénients sont assez clairs pour la tranquillité psychique : non seulement le sage ne commettra pas l'adultère, mais il se méfiera de l'amour et des relations sexuelles en général. Selon Usener ${ }^{29}$, cette doxographie reprendrait des arguments du traité Sur

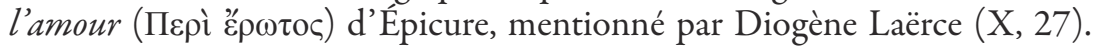
Le texte suivant est à ce sujet très clair : « ils disent qu'on n'a jamais rien gagné à l'activité sexuelle et qu'il faut même se réjouir si on n'y a rien perdu »

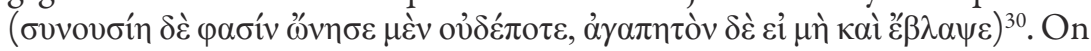
pense à la dernière phrase de la Sentence Vaticane 51 , attribuée à Métrodore : « les plaisirs du sexe, en effet, ne sont jamais profitables, et il faut se réjouir s'ils ne font pas de tort. » À une conception assez neutre de l'activité sexuelle, activité naturelle mais non nécessaire, se substitue ici une méfiance de principe à l'égard de la sexualité. T. Brennan ${ }^{31}$ propose ainsi de comprendre que le sexe n'est jamais utile et qu'on a de la chance s'il ne nous fait pas de tort. Il faudra alors sous-entendre : « or c'est ce qu'il fait habituellement. »

Un pas de plus me semble franchi au paragraphe 119 du livre X de Diogène Laërce :

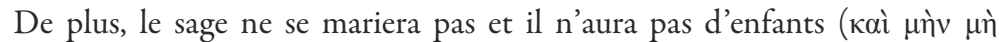

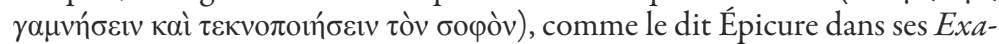
mens des apories et dans ses livres Sur la nature. Mais, en fonction des circonstances, il se mariera à un moment quelconque de l'existence.

Je retiens pour ma part le texte de l'édition Marcovich de Diogène Laërce, qui introduit une négation, à la suite de suggestions antérieures, notamment

28. Diogène Laërce, $X, 118$.

29. Usener 1887 , p. 100.

30. Diogène Laërce, $X, 118$.

31. Brennan 1996. 
de Chilton et de Brennan ${ }^{32}$. L'introduction de la négation présente plusieurs avantages. En premier lieu, elle rend mieux compte de la structure de la phrase et, ainsi, de l'opposition, bien marquée syntaxiquement, entre la position de principe et les circonstances : le sage évitera en principe de se marier, mais il pourra le faire dans des circonstances exceptionnelles et très précises, ainsi qu'Épicure, dans son testament, recommande à certains de ses disciples de le faire $^{33}$. En second lieu, dans tout ce passage, Diogène rapporte des sentences négatives, qui insistent moins sur ce que le sage fera que sur ce qu'il ne fera pas. Enfin, la correction prend en compte le texte d'Épictète, Entretiens, III, 7, 19:

Par le Dieu, imagines-tu une cité peuplée d'épicuriens ? 'Je ne me marierai pas. - Moi non plus, car il ne faut pas se marier'; ni avoir des enfants, ni s'occuper de politique! Que se passera-t-il ? D'où viendront les citoyens ? Qui les instruira?

Ce texte est particulièrement intéressant pour notre propos, car il met sur le même plan le refus du mariage et le refus de prendre part à la vie de la cité. De fait, Diogène Laërce précise que le sage épicurien, en plus d'un certain nombre d'abstentions, « ne s'occupera pas non plus de politique » (ở $\delta \grave{~}$ $\pi \mathrm{o} \imath \imath \varepsilon v ́ \sigma \varepsilon \sigma \theta \alpha \imath)^{34}$.

Tout cela témoigne d'une conception très restrictive de l'activité sexuelle, dans la perspective du 'raisonnement sobre', du calcul prudent des plaisirs et des peines, prôné par Épicure dans la Lettre à Ménécée. Les épicuriens du Jardin semblent avoir opté pour une casuistique amoureuse où le passage à l'acte, par le mariage ou hors mariage, doit faire l'objet d'une évaluation

32. Sur ce passage discuté de la doxographie éthique épicurienne, voir principalement

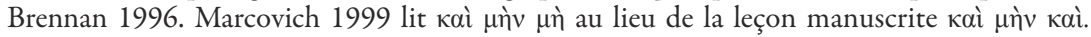
Dorandi 2013 conserve le texte des manuscrits. La correction peut être admise si l'on sousentend un verbe de défense («Épicure dit que le sage ne doit pas... »). Elle concorde par ailleurs avec Sénèque, fr. 45 Haase (Us. 19) : «Épicure, partisan du plaisir (...), dit que le sage ne doit se marier que dans de rares cas, parce qu'au mariage sont indissolublement liés de nombreux embarras. » Les épicuriens eux-mêmes ont peut-être cherché à s'opposer sur ce point au stoïcien Zénon de Citium, pour qui « le sage se mariera et engendrera des enfants » (Diogène Laërce, VII, 121). Les difficultés tiennent en partie à la nature même de l'argument épicurien : le texte discuté est extrait des Apories (Diaporiai) d'Épicure (Us. 18 à 21), ouvrage dans lequel le philosophe devait traiter les propositions de manière problématique (par arguments pour et contre). Plutarque rapporte un exemple de ce type d'analyse, exemple qu'il met au service de sa charge contre l'incivisme supposé des épicuriens : « Qưils ne faisaient pas la guerre aux législateurs, mais aux lois, on peut l'entendre de la bouche d’Épicure. Il s'interroge en effet lui-même, dans ses Apories, sur la question suivante : "le sage accomplira-t-il des actes que les lois interdisent, s'il sait qu'il ne sera pas découvert ?" et il répond : "il n'est pas facile de qualifier la chose simplement" (...) » (Plutarque, Adv. Col., 1127 D ; Us. 18). Les exceptions ou dérogations à la règle générale, comme dans le texte sur le mariage et la procréation, semblent donc justifiées par une forme de casuistique.

33. Diogène Laërce, $X, 19$.

34. Diogène Laërce, X, 119. 
rigoureuse et le plus souvent négative.

Cette attitude ne se comprend pleinement que si l'on se réfère à la conception épicurienne des émotions ou passions en général. Les épicuriens, on le sait, conçoivent les passions néfastes - émotions proprement pathologiques, par différence avec les émotions positives ou acceptables - comme des états liés au jugement faux ${ }^{35}$. Les opinions fausses sur les dieux, la mort ou le plaisir suscitent des désirs non naturels et vains. Elles pervertissent et obscurcissent les inclinations que produisent les émotions ou affections de base, à savoir le plaisir lui-même et la peine. Ces derniers pathe, en effet, sont les critères en matière de conduite, parce qu'ils indiquent naturellement ce qu'il faut éviter ou choisir ${ }^{36}$. Les passions néfastes sont à l'inverse construites à partir de jugements faux ou « vides » sur les objets susceptibles de nous faire éprouver la peine ou le plaisir, comme lorsque nous cédons au désir d'accumulation de richesses non naturelles, alors qu'il est en réalité « vide » ou « sans fondement $\gg{ }^{37}$. Les effets négatifs des passions sur l'équilibre psychique sont souvent soulignés, et cela à juste titre. Les passions aliènent littéralement le sujet, en le dépossédant de lui-même au profit de l'objet de désir (comme dans la passion amoureuse) ou de crainte (comme dans le cas de la terreur des enfers). Quant au remède, il est indiqué très clairement dans les textes dont nous disposons : il s'agit principalement de recourir à la phusiologia, la connaissance scientifique globale de la nature, pour éradiquer les illusions, maîtriser les émois passionnels et vivre en conformité avec la nature. Corrélativement, il faut se référer en toute circonstance aux critères naturels de conduite que constituent les affections de plaisir et de peine. La thérapie des passions consiste donc à savoir faire usage de nos jugements ou opinions en nous conformant aux véritables critères de vérité, notamment les sensations et les affections ${ }^{38}$, et à pratiquer le raisonnement « sobre », le juste calcul des plaisirs et des peines ${ }^{39}$.

Les épicuriens ne préconisent pas, il faut le souligner, le rejet unilatéral des passions, comme si tout état émotionnel était à proscrire. J. Procopé ${ }^{40}$, par exemple, l'a montré à propos de la colère, en soulignant que Philodème justifie une forme de colère naturelle. F. Verde a prolongé l'analyse dans cette

35. Voir notamment les analyses de Konstan 2008, p. 8-18.

36. Voir en particulier deux passages dans le préambule doxographique du livre $\mathrm{X}$ de Diogène Laërce : « dans le Canon, Épicure dit que les critères de la vérité sont les sensations et préconceptions et les affections » (Diogène Laërce, X, 31) ; « Ils disent d'autre part qu'il y a deux affections, le plaisir et la douleur, quelles sont présentes en tout être vivant, et que la première est appropriée <à sa nature>, tandis que l'autre lui est étrangère. C'est par leur intermédiaire que l'on juge en matière de choix et de refus » (Diogène Laërce, $\mathrm{X}, 34$ ).

37. $M C \mathrm{XV}, \mathrm{XXX}$.

38. Voir en particulier la $M C X X I V$.

39. Men. 130-132.

40. Procopé 1998. 
direction, en rappelant que les épicuriens ne prônent pas une éradication totale des passions, mais plutôt leur contrôle par la modération ${ }^{41}$. Certaines passions, explique F. Verde, sont tolérées chez le sage, dans la mesure où elles demeurent dans les limites définies par la nature. Cela ne veut toutefois pas dire que la même tolérance soit de mise avec la passion amoureuse, qui constitue un cas particulièrement critique de déséquilibre psychique. Le texte de Lucrèce, nous allons le voir, le confirme d'une manière on ne peut plus claire.

On retiendra donc, pour résumer ce premier temps de l'analyse, que l'activité sexuelle, à supposer qu'elle ne soit pas source de dommages, est de toute façon moralement et socialement inutile. Le fait que, selon certains épicuriens, le sage ait des raisons de s'en abstenir par principe laisse penser qu'il n'y a pas d'utilité dans la relation amoureuse. Or, pour instaurer les conditions d'une politique juste selon Épicure et Hermarque, il faut réaliser ce qui est utile à la communauté des citoyens : le juste est « quelque chose d'utile dans la vie en commun des hommes » (MCXXXVI). Enfin, la question de l'amour, dans les textes épicuriens qui semblent critiquer la sexualité en tant que telle, est considérée dans une relative indifférence à l'autre. Il s'agit fondamentalement d'un rapport à soi, évalué à partir d'un calcul sur les avantages et les désavantages. La dimension sociale de ce calcul est purement négative, lorsqu'il s'agira par exemple de ne pas contrevenir aux lois. L'essentiel est ici de maîtriser, par un travail tout intérieur et essentiellement personnel, nos propres représentations de l'objet de désir.

\section{Amour et passion dans le chant IV du poème de Lucrèce}

Examinons maintenant ce qu'il en est dans le $D R N$, et plus particulièrement dans la section «érotique » du chant IV (vers 1030-1287), où Lucrèce dépeint le désastre de la passion amoureuse. Le principe général de l'explication est bien connu : l'amant s'enchaîne à la représentation qu'il se fait de l'être aimé parce qu'il lui attribue, comme s'il s'agissait de propriétés objectives, des traits qui ne sont en fait que ses propres projections mentales. Il s'asservit à l'objet de sa passion alors que la Vénus volage, l'accouplement sans attachement, propose la voie alternative d'une jouissance libre et mutuelle. Sur le plan relationnel, on va le voir, la situation a deux caractéris-

41. Verde à paraître. Voir également Prost 2012, qui analyse les procédés épicuriens de «mesure » des affections. Dans une perspective comparable, Konstan 2007b, p. 109 sq., souligne l'importance de la joie ( $\chi \alpha \rho \alpha$ chez Épicure, laetitia chez Lucrèce) comme émotion positive éprouvée - selon lui - par la partie rationnelle de l'âme, tout en soulignant que toute joie n'est pas par elle-même justifiée ; on peut éprouver de la joie à de mauvaises pensées ou à des actions répréhensibles. Je ne me prononcerai pas ici sur l'authenticité de la distinction entre une partie rationnelle et une partie irrationnelle de l'âme, qui n'est clairement attestée, chez Épicure, que par une scholie au paragraphe 66 de la Lettre à Hérodote (Us. 311), comme le souligne notamment Verde 2010, p. 194. Voir cependant Aétius, IV, 4, 6 (Us. 312). 
tiques majeures : «l'aliénation ${ }^{42}$ consentie à l'objet de l'amour, c'est-à-dire une forme d'esclavage volontaire, et un antagonisme des partenaires qui se traduit par une violence contre nature.

La tonalité de ce passage, pris dans sa globalité, est différente de celle des textes restrictifs que j'ai évoqués précédemment. Le désir sexuel, en lui-même, est en effet la conséquence d'un état non pathologique. Il est à l'origine moralement neutre, parce qu'il est l'effet d'une nécessité proprement physiologique ${ }^{43}$. Lucrèce commence d'ailleurs par ce constat en $D R N$ II, 437, puis IV, 1030-1057, en décrivant les phénomènes de la puberté, du rêve érotique et des pollutions nocturnes. Le cas du rêve érotique est à cet égard très éclairant : ce n'est pas un simulacre de plaisir, mais un phénomène effectivement plaisant, et par conséquent un soulagement physique réel. On se réfèrera sur ce point aux observations de Diogène d'CEnoanda à propos des apparitions oniriques :

... < puisque dans le sommeil> nous atteignons effectivement les plaisirs sexuels, tout comme à l'état de veille, on ne peut pas dire que le contentement qu'elles nous procurent soit sans fondement réel sous prétexte que nous dormons. Il ne faut donc pas les dire vides, étant donné qu'elles ont un tel pouvoir ${ }^{44}$.

L'apparition en rêve de l'objet de désir peut bien être sans fondement, au sens où elle n'a pas de corrélat objectif, le plaisir n'en est pas moins réellement consistant, comme l'attestent la jouissance éprouvée et ses effets physiologiques. D'une manière générale, si l'on tient compte de la causalité proprement naturelle du plaisir, on peut dire que l'objet de l'amour est « second par rapport au processus physiologique $\gg{ }^{45}$.

Le désir sexuel, sous cette description, s'explique par un processus naturel d'expulsion, d'épuisement salutaire et cyclique, qui participe de la régulation atomique nécessaire à la vie de l'organisme. La véritable nature de l'amour est dans la régulation des humeurs ou, en termes plus généraux, des substances organiques. A. Gigandet signale à ce propos le glissement sémantique et phonétique entre amor et umor, comme en IV, 1051, où umor désigne le sang répandu sur l'ennemi qui nous a frappé. La même assonance apparaît à propos de l'opposition entre la substance (umor) gardée dans le corps - liquide qu'il convient d'évacuer dans un autre corps - et la conservation douloureuse de ce même liquide, quand un amour (amor) exclusif nous

42. Pour reprendre l'expression de J. Salem, dans Salem 1990, p. 172 sq.

43. Le point de départ de l'amour (la puberté) et ses effets doivent être analysés comme des phénomènes physiologiques, par exemple en termes d'abcès ou de blessures, ainsi que le souligne Baier 2010, p. 98-99. Sur la dimension physiologique de la section finale du chant IV, voir encore Schrijvers 1999, p. 129, qui établit que cette section est liée à un ensemble de considérations sur les fonctions vitales; Landolfi 2013, p. 23-39.

44. Diogène d'OEnoanda, fr. 10 IV 2-6 Smith.

45. Pour reprendre les observations de Gigandet 1999, p. 83. 
dicte de le réserver à un partenaire unique (IV, 1063-1067). Lucrèce voit à l'évidence, dans le mécanisme articulant l'entrée des images sensorielles à l'éjaculation (1030-1038), une économie organique naturelle, comparable à celle de la respiration, qui fonctionne selon un principe de compensation entre entrée et sortie ${ }^{46}$. Jusqu'ici, nous sommes face à des phénomènes strictement naturels, comme le désir de boire quand la chaleur à l'intérieur de l'estomac est excessive ${ }^{47}$.

Lucrèce n'est pas pour autant en désaccord avec Épicure sur l'idée selon laquelle le désir sexuel est naturel mais non nécessaire : on peut supposer que, au-delà de la nécessaire régulation physiologique des humeurs, la relation amoureuse et l'interaction sexuelle avec un partenaire ne sont ni vitales ni indispensables à l'équilibre psychique. Le fait d'en être privé n'est pas nécessairement douloureux, et le trop-plein d'humeurs peut être évacué par d'autres moyens.

Quoi qu'il en soit, la neutralité de l'analyse lucrétienne, quand elle se place du strict point de vue physiologique, tranche avec les expressions de méfiance repérées plus haut dans la doxographie éthique des premiers épicuriens. L'éloge de l'amour libre - et, peut-on penser, naturel -, en $D R N$, IV, 1141 sq., viendra confirmer la neutralité morale de la sexualité bien comprise, une forme d'amour qu'il ne s'agit, ni de recommander comme s' il était nécessaire, ni de condamner comme s'il devait fatalement nous troubler, malgré les mises en garde du Maître du Jardin à ce sujet ${ }^{48}$. Corrélativement, l'invitation à se tourner vers un autre corps que celui de l'être aimé est avant tout thérapeutique : il s'agit, quand on éprouve les douleurs liées au sentiment amoureux, de « fuir les images » (IV, 1064), et, pour y mieux parvenir, de détourner physiologiquement notre substance vers un autre objet de désir. La pura voluptas ${ }^{49}$ de la sexualité sans attaches n'est valorisée que par opposition aux ravages de la passion. Assurément, Lucrèce évoque la sincérité et la réciprocité du désir (IV, 1192-1200), mais cela ne fait pas pour autant du plaisir sexuel un bénéfice devant être recherché pour lui-même. L'incitation aux joies de l'amour est, on le reconnaîtra, minimale, quand Lucrèce engage simplement à « en recueillir les bienfaits sans payer de rançon » (IV, 1074).

46. $D R N$, IV, 937-938.

47. DRN, IV, 870-876. Konstan 2007a, p. 96-98, établit un parallèle entre ce passage et les vers IV, 1086-1087, sur le feu inextinguible de la passion amoureuse, entretenu par une extrapolation sans fondement.

48. Brown 1987, p. 121-122, considère que Lucrèce concorde parfaitement avec Épicure, et ne diffère du Maître que dans les moyens d'expression. Il estime cependant très probable que Lucrèce est, de tous les épicuriens de l'Antiquité, le seul à avoir relié la question du sexe à la problématique de l'illusion. Je pense pour ma part que cette caractéristique du propos lucrétien s'accompagne d'un second trait spécifique : l'idée que l'activité sexuelle n'a de valeur qu'en fonction de l'attitude que nous adoptons face aux passions.

49. Expression utilisée par exemple en $D R N$, IV, 1075. 


\section{Sexualité et société dans le chant $\mathrm{V}$}

L'opposition entre désir naturel et passion amoureuse, parce qu'elle implique la relation à autrui, va précisément conduire Lucrèce à croiser les deux thèmes qui nous occupent, celui de l'amour et celui de la politique ou, plus généralement, de la sociabilité. Pour observer ce tournant, il faut partir de la généalogie lucrétienne des sociétés au chant $\mathrm{V}$. On note en premier lieu qu'il n'y a pas de véritable continuité entre l'association naturelle des partenaires sexuels et la constitution des liens affectifs puis des rapports sociaux. Tout au plus peut-on voir là une succession hasardeuse d'étapes accidentelles.

Au début de la généalogie du chant $V$ (v. 925-1010), Lucrèce invite à se représenter la vie des premiers hommes. Le passage le plus significatif pour mon propos - je souligne les termes essentiels à son analyse - est le suivant :

Le bien commun, ils ne pouvaient l'envisager, et rien, ni coutume ni loi, ne réglait leurs rapports. Ils saisissaient toute proie que le hasard leur offrait, ayant appris à vivre et prospérer chacun pour soi. Et Vénus dans les bois unissait les corps des amants. Nulle qui ne cédât au désir partagé, ou à la vive violence et à l'élan de l'homme en rut, ou au gain, arbouses, baies et poires choisies.

Nec commune bonum poterant spectare, neque ullis

moribus inter se scibant nec legibus uti.

Quod cuique obtulerat praedae fortuna, ferebat

sponte sua sibi quisque valere et vivere doctus.

Et Venus in silvis iungebat corpora amantum;

conciliabat enim vel mutua quamque cupido

vel violenta viri vis atque inpensa libido

vel pretium, glandes atque arbita vel pira lecta ${ }^{50}$.

Je retiendrai de ce tableau primitif trois caractéristiques :

(a) Les premiers hommes mènent la vie des bêtes et ne connaissent pas les techniques; leur préservation est garantie par la nature. Ils pratiquent la chasse (v. 966-972).

(b) Ils ignorent le « bien commun » (commune bonum - v. 958); il n'y a « pas de coutumes ni de lois » qui organisent la vie commune. Ils sont essentiellement instruits par le hasard (fortuna - v. 960), par le spectacle de la nature (v. 961) et par l'expérience et l'habitude (v. 977).

(c) Le désir sexuel est un principe d'association minimale, réunion parfois motivée par un « désir partagé », mais qui est aussi, dans d'autres cas, violente ou négociée (v. 962-963).

L'union des partenaires sexuels est donc une association pré-sociale, qui s'oppose à l'union fondée sur le « bien commun », c'est-à-dire au lien

50. DRN, V, 958-965 (trad. Kany-Turpin 1997). 
construit, légalisé ou tout au moins réglé, des communautés organisées et, plus tard, des cités. C'est seulement dans un deuxième temps ${ }^{51}$, étape bien marquée par un indicateur temporel (inde: V, 1011), et à partir d'une union plus constante entre l'homme et la femme (et mulier conjuncta viro) ${ }^{52}$, que l'habitat induit la première forme de sociabilité organisée, fondée sur l'amitié et le souci de protéger les plus faibles. Apparaissent alors les premiers « pactes » (foedera) nécessaires à la préservation de l'humanité (v. 10251027). Viennent à la suite l'affection pour la progéniture et les liens d'amitié, puis un respect des « pactes » ou « contrats » (foedera) entre membres de l'espèce humaine $(\mathrm{V}, 1025)$. Nous ne sommes pas encore à l'état civil le plus élaboré, mais le mode de régulation des relations est radicalement différent de celui de la première période.

Je suis pour cette raison en net désaccord avec la lecture « continuiste » défendue par M. Nussbaum. Cette interprétation repose sur deux options selon moi très discutables ${ }^{53}$. La première consiste à affirmer que la vie des premiers hommes est bien plus enviable que la nôtre (« this is a far better life than ours $\gg$ ). En réalité, ses avantages sont décrits de manière essentiellement négative, par pur contraste avec les perversions de la vie civile, et Lucrèce ne nous invite pas à revenir à un tel état. La nature primitive que Lucrèce retrouve en écrivant une préhistoire de l'humanité n'est pas une valeur en soi et n'appelle à aucun « primitivisme ${ }^{54}$. L'idéal de vie auquel il invite Memmius, pour naturel qu'il soit, requiert tout l'art de la discipline épicurienne, art particulièrement sophistiqué. Il est vrai que pour les épicuriens de la période, pour Philodème notamment, la modération des passions suppose une distinction entre affect naturel et affect non naturel (comme dans l'opposition philodémienne entre colère pathologique et colère naturelle), ainsi qu'on l'a vu plus haut. Cela ne signifie pas pour autant qu'en se conformant à la nature le sage revienne à la nature primitive, à supposer qu' un tel retour soit possible. Son rapport à la nature, comme dans la préconisation lucrétienne de liberté amoureuse, suppose une éducation à la fois mentale et éthique,

51. Comme le montre Algra 1997.

52. $D R N, \mathrm{~V}, 1012$. Il n'est pas impossible que le terme conjuncta, qui s'applique naturellement à la relation conjugale, fasse ici écho à la distinction ontologique entre les « propriétés » (conjuncta) inséparables de leur substrat (comme le poids pour la pierre ou la fluidité pour l'eau) et les « accidents » (eventa), comme l'esclavage ou la liberté, la pauvreté ou la richesse (DRN, I, 451-458). Si l'on retient cette hypothèse, on comprend mieux encore la différence entre le tout premier stade de la vie humaine, où les unions sont purement accidentelles et hasardeuses, et celui, postérieur, des unions stabilisées par les règles sociales naissantes.

53. Nussbaum 1994, p. 162. Pour une critique de cette interprétation, voir déjà Gigandet 2003 et Landolfi 2013, p. 191-203, selon qui l'union stable fondée sur l'habitude ne saurait constituer qu'une possibilité circonstancielle d'apaisement des passions, et non pas une solution constante au problème, à la fois psychologique et social, de la passion amoureuse.

54. Voir en ce sens Campbell 2003, p. 10-12. 
ainsi qu'une forme élaborée de rationalité qui n'a rien de commun avec la vie des premiers hommes. Le naturel, pour les épicuriens, ne se réduit pas à l'originel.

La seconde option de M. Nussbaum consiste à donner une justification téléologique au processus de développement des sociétés, comme si Vénus accompagnait un plan inscrit dans la nature de l'être humain. Je cite M. Nussbaum :

he also makes it very clear that this life is not a complete life, this Venus not a complete Venus, for human beings; that human affection and desire must evolve in order to include the tenderness toward others, the concern for laws, institutions, and the common good, that are essential for truly human happiness.

Cette idée d'un état « incomplet » qui appellerait une « évolution » vers les liens affectifs, ainsi que vers le respect des lois, des institutions et du bien commun me paraît en contradiction directe, non seulement avec la tonalité, pour le moins réservée, des propos de Lucrèce sur la politique, mais également - et même plus encore - avec la critique de la finalité naturelle que l'on trouve au chant IV (825-857). La Kulturgeschichte lucrétienne, sa description des « progrès » de l'humanité, met d'ailleurs au premier plan le hasard et les circonstances ${ }^{55}$, comme en témoigne, parmi d'autres exemples, la découverte fortuite de l'usage du feu grâce à la chute de la foudre (V, 1091-1107).

La suite du texte du chant $\mathrm{V}$ va du reste montrer, avec l'apparition des rois, puis de l'oppression et de la violence guerrière (V, 1108-1160), que les institutions sociales et politiques jouent un rôle fondamentalement ambigu dans cette histoire : tout en étant devenues nécessaires à la préservation des hommes, elles favorisent de nouvelles formes de discorde et d'oppression en suscitant de nouvelles craintes et des désirs illimités. On comparera ce phénomène à l'évocation finale de la Peste d'Athènes (VI, 1138-1286), où la peur de la mort nourrit une panique collective - constituant ainsi un fait collectif pathologique - et dissout les liens sociaux en supprimant toute solidarité entre les individus ${ }^{56}$. Dans ces cas extrêmes, les comportements grégaires, associations fondées sur l'urgence et la terreur, sont à l'opposé d'une sociabilité positive capable de réaliser, comme le préconisait Épicure, une vie juste, c'est-à-dire conforme à l'utilité commune. En $D R N$, III, 31-78, la violence de la lutte pour le pouvoir, la richesse et les honneurs s' inscrivent dans cette même ligne de discours négatif sur la politique. Là encore, le rapport à autrui conduit en fait à sa négation même, puisque la peur de la mort qui soustend cette conduite « rompt les liens d'amitié » (vincula amicitiai rumpere: III, 83-84). D’une manière générale, dans le récit de Lucrèce, l'organisation politique, le pouvoir et les lois n'apparaissent que dans le contexte d'une exis-

55. Comme le montre Schiesaro 2007, p. 45, qui qualifie le récit de l'évolution des sociétés de « rigorously non-teleological and non-linear account ».

56. Voir VI, 1239-1242; 1280-1281. 
tence coupée de la nature et pervertie par la vie sociale ${ }^{57}$.

La rupture du chant $\mathrm{V}$ marque en tout cas un point de non-retour dans l'histoire de l'humanité. Les lois ne sont pas un mal en soi, mais elle ne se conçoivent dans ce contexte que par opposition à la vie des premiers hommes, qui n'était pas réglée par des rapports de pouvoir ou des contrats. La sexualité primitive, évoquée au premier stade de la généalogie lucrétienne, avant la découverte des techniques et des règles de sociabilité, est d'autant plus importante qu'elle constitue le seul principe de rapport à autrui. L'accouplement répond à un désir immédiat, qui ne differe pas, fondamentalement, des autres formes d'appétit $(\mathrm{V}, 962-965)$. Lucrèce indique que chacun vit « pour soi, selon sa propre décision » $(\mathrm{V}, 961)$, ce qui suggère que les relations sexuelles n'ont alors d'autre mobile que le désir propre de chacun. Le désir sexuel, en l'occurrence, apparaît comme la conséquence comportementale immédiate du besoin physiologique, voire comme purement alimentaire, si l'on considère qu'il est mis sur le même plan que l'appétit pour les aliments. La sexualité primitive, que Lucrèce ne présente visiblement pas comme un modèle à suivre mais comme un pur fait, témoigne simplement de la possibilité d'associations humaines minimales en dehors des règles sociales.

\section{Passions érotiques et passions politiques}

Revenons enfin au chant IV et aux passages qui invitent à croiser la passion amoureuse et les vaines passions liées à la politique. En premier lieu, Lucrèce établit une analogie très précise entre les maux de l'amour et ceux qu'engendre la société, alors même, comme on l'a vu, que l'amoureux passionné en oublie ses devoirs sociaux (officia) et sa réputation (fama) (IV, 1124).

Sur ce point, D. Fowler, dans le passage de son article sur la politique lucrétienne où il évoque la sexualité, le fait de manière très instructive. Il établit que Lucrèce, lorsqu' il évoque Sisyphe (III, 995-1002), montre qu'il est comparable au politicien qui aspire sans cesse au pouvoir sans jamais l'obtenir $^{58}$. Or les termes utilisés sont aussi ceux qui servent à décrire la soif inextinguible de l'amoureux passionné : la quête du pouvoir « est vaine » (inane est), et celui qui la conduit imbibit, c'est-à-dire : «s'acharne à briguer », « prétend à... », mais aussi « boit », « se pénètre de ». Parallèlement, l'amant est comparé au dormeur dévoré par la soif, et se voit mourir de soif au fond du torrent où il boit (IV, 1097-1100), exemple frappant d'un désir vain.

Plus nettement encore, le politicien ambitieux ressemble trait pour trait, dans l'exercice de la démagogie, à l'amant aliéné à l'objet de son amour, comme le Calliclès du Gorgias platonicien, amoureux d'un individu

57. On comprend bien par cet exemple l'influence que le $D R N$ a pu avoir sur le Discours sur l'origine et les fondements de l'inégalitéparmi les hommes.

58. Cela ne veut pas dire que le pouvoir politique, l'imperium, soit lui-même vide de tout contenu et de tout sens, ainsi que l'a montré Fish 2011. 
dénommé Démos comme du démos d'Athènes ${ }^{59}$. Pour Lucrèce, l'amour passionnel nous enchaîne aux volontés de l'aimé (IV, 1122) et ceux qui se veulent puissants sont frappés par l'invidia comme par la foudre (V, 11261127).

De même, dans le prologue du chant II, l'agitation de ceux que l'on observe de loin, comme on regarde du rivage les flots tourmentés, se traduit par une errance infinie : « on peut voir les autres errer (errare) sans trêve en bas, cherchant le chemin de la vie, rivalisant de talent, de gloire nobiliaire » (II, 9-11). Or les regards insatiables de l'amoureux « errent incertains sur le corps tout entier » (errantes incerti corpore toto: IV, 1104). Au vers 1077, l'ardeur des amants est prise dans des errances incertaines (fluctuat incertis erroribus ardor amantum). L'incertitude qui caractérise l'agitation des cités est de même ordre que l'errance amoureuse. Les amants « aveugles de désir » (cupidine caeci : IV, 1153) font écho au « désir aveugle des honneurs » (bonorum caeca cupido : III, 59) qui pervertit la vie commune.

Le chant IV suggère que l'union immédiate, l'amour libre, est le meilleur remède aux troubles suscités par l'amour passionnel, parce qu'il restaure l'équilibre des humeurs, Lucrèce jouant à ce propos de l'assonance umor amor, déjà signalée :

Il convient de fuir sans cesse les images, de repousser ce qui peut nourrir notre amour, de tourner ailleurs notre esprit et de jeter en toute autre personne le liquide amassé, au lieu de le garder, au même amour voué, et de nous assurer la peine et la souffrance.

Sed fugitare decet simulacra et pabula amoris

absterrere sibi atque alio convertere mentem

et iacere umorem coniectum in corpora quaeque

nec retinere semel conversum unius amore

et servare sibi curam certumque dolorem ${ }^{60}$.

Il montre également que l'union naturelle des sexes, telle qu'on peut l'observer chez les animaux et chez les hommes, ne repose originellement sur aucun autre principe que le «plaisir commun » (communis voluptas) ou la « joie mutuelle » (communa gaudia; mutua gaudia) (IV, 1195-1208). Les hommes primitifs, on l'a vu, ne connaissent pas le bien commun (commune bonum : V, 958). Ce qui est commun, dans l'amour libre comme dans les unions primitives ou animales, ce n'est pas le bien compris comme valeur sociale, mais le désir de s'accoupler et le simple plaisir physique, c'est-àdire un bien strictement naturel. L'opposition est d'autant plus claire que

59. Voir DRN, V, 1131-1135 et Platon, Gorgias, 481e. Le genre humain, en acceptant les lois, « se soumet de lui-même » $(\mathrm{V}, 1146-7)$, tout comme l'amant se soumet aux désirs et à sa propre représentation de l'être aimé.

60. DRN, IV, 1063-1067 (trad. Kany-Turpin). 
communis apparaît trois fois dans le passage du chant IV sur la réciprocité du désir et du plaisir. À ce plaisir commun, qui n'est pas dépourvu d'une certaine violence naturelle, s'oppose la violence contre nature qui caractérise l'amour passionnel : « leur proie, ils l'étreignent à lui faire mal, morsures et baisers lui abîment les lèvres » (IV, 1079-1080).

L'usage de communis dans les deux textes, celui du chant IV et celui du chant V, est particulièrement significatif, car l'adjectif a toujours, chez Lucrèce, un sens fort. On recense vingt occurrences ${ }^{61}$, qui se laissent clairement classer dans des rubriques bien définies :

(1) un sens politique : bien commun ( $\mathrm{V}, 958)$, mal commun provoqué par la guerre $(\mathrm{V}, 1343)$, pacte commun de la paix $(\mathrm{V}, 1155)$, absence de remède commun contre la peste (VI, 1226);

(2) un sens physique : les atomes sont le corps commun des choses, comme les lettres de l'alphabet pour l'écriture (I, 196, 896, 824; II, 689, 692) ; à propos de l'âme et du corps (III, 325, 335), de la Terre et du Ciel (V, 554).

Il n'est donc pas indifférent que Lucrèce utilise systématiquement un terme chargé d'un sens aussi fort, qu'il soit politique ou physique. Le fait que le plaisir éprouvé dans la sexualité naturelle soit commun et mutuel invite clairement à admettre une forme d'association minimale et spontanée, antérieure à tout contrat, ainsi qu'une activité commune sans aliénation d'une partie à l'autre. L'accord est naturel et non pas extorqué, selon le principe du bénéfice respectif des deux parties. La suite du texte traite d'ailleurs de la reproduction selon un principe déjà présent chez Démocrite ${ }^{62}$, qui veut qu'il y ait concurrence entre deux semences, mâle et femelle. Cette concurrence est dite ici « harmonieuse », « sans vainqueur ni vaincu » (IV, 1217) entre homme et femme. Ce passage confirme l'égalité de statut entre les géniteurs : c'est « l'ardeur mutuelle » (mutuus ardor, 1216) qui, « par conspiration » (conspirans), permet la reproduction. La fertilité est affaire d'harmonia (1248). À l'inverse, l'amour passionnel est déséquilibré, et sa violence est liée à une attitude de domination imposée. Comme le dit Brown à propos de cette opposition, il s'agit d'un acte de violence unilatéral et désespéré63. Le plaisir mutuel est donc un lien naturel, non seulement antérieur à toute convention politique, mais encore en fort contraste avec les métaphores guerrières qui servent à illustrer l'agressivité de l'amour passionnel. Ces dernières font précisément écho à la violence des situations politiques et aux évocations de la guerre elle-même - peut-être aux conflits qui agitent la République du temps de Lucrèce -, comme dans l'hymne à Vénus du chant I, où la Déesse apporte la paix dans une étreinte avec le dieu Mars (I, 21-43), étreinte dont

61. Wacht 1991. Brown 1987 souligne, à propos des occurrences que l'on trouve en IV, 1195-1207, leur caractère à la fois emphatique et récurrent.

62. Voir notamment Aristote, Génération des animaux, IV, 1, 764a6-11 [DK 68 A 143].

63. «A one-sided and desperate act of near violence », Brown 1987, p. 66 . 
la nature exacte reste peut-être à préciser ${ }^{64}$, mais qui exprime en tout cas une affection littéralement désarmante.

Aux conventions politiques et juridiques, on doit associer les conventions sociales qui régissent indirectement les comportements amoureux passionnels. En se laissant conduire par une sexualité de conventions, telle que l'imaginaire littéraire la reproduit et la suscite, l'amoureux passionné renonce plus encore à sa liberté. On le voit bien dans le passage consacré aux parfums. Les odeurs en elles-mêmes sont perçues par le biais d'affections non passionnelles, d'affects sensoriels qui, comme les autres sensations, sont globalement fiables. Ainsi, dans le long passage du chant IV (673-705) que Lucrèce consacre à l'odorat, les odeurs sont appréhendées comme de simples indicateurs des propriétés de l'objet. L'odeur du miel attire les abeilles et celle des cadavres attire les vautours. Les odeurs orientent, et même « guident » (ducit : IV, 685) la conduite. Les oies du Capitole reconnaissent l'odeur humaine à distance et ne se trompent pas. L'odeur, prise en elle-même, est neutre et dépourvue d'élément affectif ${ }^{65}$. Il en va tout différemment quand elle est associée à un objet d'amour passionnel :

La malheureuse exhale une odeur repoussante, ses servantes s'enfuient et se rient en cachette, mais l'amant éconduit pleurant devant sa porte souvent couvre le seuil de fleurs et de guirlandes, parfume de marjolaine les montants implacables et plante ses baisers, pauvre homme, au cœur du bois! Toutefois, s'il était reçu, dès le premier effluve il chercherait pour fuir une excuse honorable. Adieu l'élégie profonde et longtemps méditée : il maudirait sa sottise d'avoir à la belle prêté plus qu'il ne convient d'accorder à une mortelle. Et nos Vénus le savent bien, qui mettent tant de soin à toujours dérober les coulisses de leur vie aux amants qu'elles veulent maintenir enchaînés.

Et miseram taetris se suffit odoribus ipsa, quam famulae longe fugitant furtimque cachinnant. At lacrimans exclusus amator limina saepe floribus et sertis operit postisque superbos unguit amaracino et foribus miser oscula figit; quem si iam ammissum venientem offenderit aura una modo, causas abeundi quaerat honestas et meditata diu cadat alte sumpta querella stultitiaque ibi se damnet, tribuisse quod illi plus videat quam mortali concedere par est. Nec Veneres nostras hoc fallit; quo magis ipsae omnia summo opere hos vitae postscaenia celant, 
quos retinere volunt adstrictosque esse in amore ${ }^{66}$.

Lucrèce illustre ici l'aliénation amoureuse en la confrontant, par une sorte d'exercice de pensée, à l'odeur objective de l'être aimé. L'amant ne la perçoit pas et croit aimer véritablement, mais ses élans cesseraient aussitôt s'il était exposé aux odeurs qu'on s'emploie à lui cacher. Brown doute qu'il s'agisse de l'odeur naturelle et pense plutôt à des fumigations médicales ${ }^{67}$, tandis qu'un passage de l'Art d'aimer d'Ovide inviterait plutôt à songer aux odeurs de maquillage. Ovide conseille d'ailleurs de se maquiller en secret ${ }^{68}$. On comprend en tout cas, précise Lucrèce (avec une évidente ironie ${ }^{69}$ qui dénonce les termes mêmes qu'il utilise), que certains aspects de la « vie » réelle, c'est-à-dire sans doute « corporelle », soient savamment gardés dans l'arrière-scène (postscaenia), tandis que la toilette ou les parfums, peut-on penser, occupent la scène corporelle, faisant écran devant l'intime. Brown signale en effet que la scaena, vers la fin de la République, était souvent décorée. Les ornements de la scène s'opposent implicitement au désordre des coulisses. En d'autres termes, l'amant n'est amoureux que par représentation, en projetant sur l'objet du désir ses propres constructions mentales, constructions qui révèleraient leur vacuité si de nouvelles images plus fortes - celles que feraient naître des odeurs repoussantes - venaient les contrarier. Toutefois, au-delà du phénomène de projection - caractéristique, on l'a rappelé, de la passion en général et de la passion amoureuse en particulier -, l'attitude de l'amant éconduit a une autre dimension, conventionnelle et sociale. La scène de la plainte amoureuse devant la porte de l'être aimé est en effet un motif classique dans la tradition littéraire de l'élégie romaine ${ }^{70}$. L'expression, ici fortement théâtralisée, de la douleur amoureuse, montre à quel point l'amour passionnel et les jeux de séduction dépendent de modèles comportementaux codifiés par des conventions à la fois sociales et littéraires $^{71}$. Alors que la sexualité primitive et celle qu'inspire la Vénus volage unissent les individus sans aucun rituel social, la passion amoureuse dicte des comportements stéréotypés, régis par des normes collectives factices.

L'évocation de l'amour conjugal (IV, 1278-1287), enfin, semble offrir une solution thérapeutique supplémentaire aux dangers de l'amour-passion :

66. $D R N$, IV, 1175-1187 (trad. J. Kany-Turpin).

67. Brown 1987, p. 296.

68. Ovide, Ars amat., III, 213. On m'a d'ailleurs signalé qu'il serait intéressant, en complément de la présente analyse, de comparer la dénonciation lucrétienne de l'illusion amoureuse à la manière dont Ovide justifie le libertinage et l'artifice.

69. Brown 1987, p. 303.

70. Voir encore Brown 1987, p. 298-299. Sur le contexte littéraire du traitement lucrétien de l'amour dans son ensemble, voir Landolfi 2013.

71. Il faut y adjoindre les stéréotypes liés à la religio, comme l'image des flèches que tireraient les divinités pour provoquer le sentiment amoureux. Cette représentation est en effet moquée par Lucrèce en IV, 1278-1279. 
l'habitude (consuetudo) et une stable coexistence ont la force de la constance, comme les gouttes d'eau qui finissent par user la pierre, tout à l'opposé de la fureur passionnelle. Cependant, la brièveté et le caractère allusif du passage ne permettent pas de se faire une idée claire du rôle de la vie conjugale dans la thérapie des passions. M. Nussbaum soutient pourtant une thèse assez audacieuse à ce sujet, voyant dans l'amour conjugal le véritable remède à la passion destructrice : « the goal of Lucretian therapy is to make a good marriage possible $»^{72}$. Cette solution $\mathrm{du}$ 《bon mariage », éminemment sociale, ne peut toutefois occulter cet autre rapport à la politique que je viens de souligner : c'est avant tout contre la vie en cité et contre la participation active à la politique, contre la guerre et contre les normes sociales (y compris les codifications comportementales et littéraires de l'amour), que se construit le modèle lucrétien de l'amour libre. De ce point de vue, l'amour conjugal ne constitue que le versant socialement acceptable, alternatif par rapport à la voie subversive et radicale de l'union physique sans attache, de la thérapie des émotions amoureuses.

Si l'on considère que l'éloge de la Vénus volage du chant IV ne décrit pas les hommes primitifs et, en ce sens, ne prône pas une union purement naturelle, mais plutôt une manière naturelle de pratiquer l'amour, on voit que la sexualité sans attachement est, pour Lucrèce, une option toujours actuelle, un choix possible, et donc une réponse pratique et thérapeutique aux excès de l'amour passionnel. D'une manière générale, le message de Lucrèce est clair : on peut désirer autrement que par passion, et sans reproduire en amour la manière dont les hommes publics, en politique, désirent le pouvoir. L'amour libre est en ce sens une justification supplémentaire, contre les attraits superficiels de la vie publique, du choix épicurien de « vivre caché ».

Il me semble donc que l'évocation de l'amour primitif, en un sens, mais plus encore la justification de l'amour volage, sont non seulement une alternative radicale à l'amour passionnel, mais encore un contre-modèle fécond face à cette autre forme d'union que constitue la vie sociale et face aux vaines ambitions qu'entretient la pratique politique. À la communauté du plaisir, fondée sur le seul principe du bénéfice mutuel, s'opposent corrélativement la passion dévastatrice et la sociabilité aliénée des cités corrompues. Je ne crois pas que l'amour doive, pour Lucrèce, remplacer la politique, ni fonder le lien social. Rien ne le laisse entendre dans les textes que nous avons parcourus. Il ne s'agit pas non plus de revenir à un pur et simple état de nature dans une perspective utopique ou primitiviste. L'amour sans attachement a toutefois une triple utilité : l'utilité physique qu'il y a à satisfaire ses besoins physiologiques, l'utilité psychique que nous trouvons à éviter les troubles de l'amour passionnel, l'utilité psychique encore d'une activité libérée du lien «politique ».

72. Nussbaum 1994, p. 185. 
Ce type de relation ne fait pas l'objet de recommandations directes ; il ne constitue pas une norme pratique susceptible d'être intégrée telle quelle à la doctrine éthique du Jardin. S'il en allait ainsi, cela placerait d'ailleurs Lucrèce en contradiction avec les restrictions formulées par Épicure à propos de la sexualité. L'évocation de la voie alternative d'un amour conjugal paisible suffit à montrer le caractère circonstanciel de l'éloge de l'amour volage. Comme on l'a dit plus haut, la sexualité sans attaches n'est valorisée que par opposition aux ravages de la passion, et non pas pour elle-même. La représentation d'une telle relation est en tout cas pleinement légitime aux yeux de Lucrèce, à titre de contre-modèle : le discours sur l'amour et le contraste qu'il institue entre sexualité libre et amour passionnel participent directement de la thérapie de l'âme, face aux dangers de la passion et des appétits de domination. Il me paraît ainsi très probable que, pour Lucrèce, le remède à la passion amoureuse exerce également ses vertus sur les différentes pathologies associées, parmi lesquelles les désirs vains qui accompagnent la politique et le pouvoir.

Que conclure enfin sur Lucrèce et la politique ? Il est difficile, on l'a vu, de donner une réponse tranchée ou univoque. Quoi qu'il en soit, l'angle d'analyse que j'ai adopté, le croisement de l'amour et de la politique, ne peut que laisser le lecteur dubitatif face à la possibilité d'une politique harmonieuse et juste. En proposant une alternative à la passion amoureuse, et en rejetant ce qui, dans la sphère de l'intime, reproduit l'oppression et l'aliénation qui caractérisent le vain appétit de pouvoir, Lucrèce dénonce les mêmes passions qui, tout à la fois, dévorent les amants et corrompent les cités. 


\section{BIBLIOGRAPHIE}

Algra, K. A. 1997 : « Lucretius and the Epicurean Other : On the Philosophical Background of $D R N$ V.1011-1027 », dans Algra, K. A., M. H. Koenen, P. H. SCHRIJVERS 1997 (éd.), p. 141-150.

Algra, K. A., M. H. Koenen, P. H. Schrijvers 1997 (éd.) : Lucretius and his Intellectual Background, Amsterdam, 1997 (Verhandelingen der Koniklijke Akademie van Wetenschappen, 172).

BAIER, Th. 2010 : « Das Irrationale bei Lukrez », Würzburger Jahrbücher für die Altertumswissenschaft, 34 (2010), p. 97-114.

DOI : https://doi.org/10.11588/wja.2010.0.32534

Brennan, T. 1996 : « Epicurus on Sex, Marriage and Children », Classical Philo$\log y, 91 / 4$ (1996), p. 346-352.

DOI : https://doi.org/10.1086/367525

Brown, E. 2009 : «Politics and Society », dans J. Warren (éd.), The Cambridge Companion to Epicureanism, Cambridge, 2009 (The Cambridge Companions to Philosophers), p. 179-196.

BRown, R. D. 1987 : Lucretius on Love and Sex : a commentary on De rerum natura IV, 1030-1287, Leiden, 1987 (Columbia Studies in the Classical Tradition, 15).

Campbell, G. L. 2003 : Lucretius on Creation and Evolution. A Commentary on De Rerum Natura, Book Five, Lines 772-1104, Oxford, 2003 (Oxford Classical Monographs).

Dorandi, T. 2013 (éd.) : Diogenes Laertius, Lives of Eminent Philosophers, Cambridge, 2013 (Cambridge Classical Texts and Commentaries, 50).

FABRE-SERRIS, J. 2007 : « La notion de voluptas chez Lucrèce et sa réception dans la poésie érotique romaine (Virgile, Buc. 2 ; Géorg. 3 ; Properce, 1, 10 ; Ovide, Hér. 18 ; Ars ; Mét., 4) », dans L. Boulègue, C. Lévy (éd.), Hédonismes. Penser et dire le plaisir dans l'Antiquité et à la Renaissance, Villeneuve d'Ascq, 2007 (Cahiers de philologie, 23. Série Apparat critique), p. 141-159.

DOI : 10.4000/books.septentrion.9291.

Fohlen, G. \& J. Humbert 1931 (éd. et trad.) : Cicéron, Tusculanes, 2 vol., Paris, 1931 (Collection des universités de France. Série latine).

Fowler, D. P. 1989 : « Lucretius and politics », dans M. Griffin \& J. Barnes (éd.), Philosophia togata : [1] Essays on Philosophy and Roman Society, Oxford, 1989, p. 120-150.

FISH, J. 2011 : « Not all Politicians are Sisyphus : What Roman Epicureans were Taught about Politics », dans J. Fish \& K. Sanders (éd.), Epicurus and the Epicurean Tradition, Cambridge, 2011, p. 72-104.

Gigandet, A. 1999 : « De l'amour. Vénus de Lucrèce \& Erôs platonicien », dans R. Poignault (éd.), Présence de Lucrèce. Actes du colloque tenu à Tours (3-5 décembre 1998), Tours, 1999 (Caesarodunum, 32b), p. 77-85.

GigandeT, A. 2003 : « Lucrèce et l'amour conjugal : un remède à la passion ? », dans B. Besnier, P.-F. Moreau, L. Renault (éd.), Les passions antiques et médiévales, Paris, 2003, p. 95-110.

DOI : 10.3917/puf.bedou.2003.01.0095

Giovacchini, J. 2014 : « La perception érotique du corps dans l’esthétique épicu- 
rienne », dans G. Puccini (éd.), Le débat des cinq sens de l'Antiquité à nos jours, Pessac, 2014 (Eidôlon, 109), p. 91-100.

Gordon, P. 2012 : The Invention and Gendering of Epicurus, Ann Arbor, 2012.

Kany-Turpin, J. 1997 (éd. et trad.) : Lucrèce, De la nature, éd. revue, Paris, 1997 (GF, 993).

Koenen, M. H. 1997 : « Lucretius' Olfactory Theory in De rerum natura IV », dans Algra, K. A., M. H. Koenen, P. H. Schrijvers 1997 (éd.), p. 163-177.

Konstan, D. 2007a : Lucrezio e la psicologia epicurea, trad. it. I. Ramelli, Milan, 2007 (Studi e Testi, 103).

Konstan, D. 2007b : « L'âme », dans A. Gigandet, P.-M. Morel (éd.), Lire Épicure et les épicuriens, trad. fr. J. Giovacchini, Paris, 2007 (Quadrige), p. 98-116.

Konstan, D. 2008 : A Life Worthy of the Gods. The Materialist Psychology of Epicurus, Las Vegas/Zurich/Athens, 2008.

LANDolfi, L. 2013 : Simulacra et pabula amoris. Lucrezio e il linguaggio dell'eros, Bologna, 2013 (Testi e manuali per l'insegnamento universitario del latino, 127).

Laurand, V. 2005 : La Politique stoücienne, Paris, 2005 (Philosophies, 185).

Laurand, V. 2014 : Stö̈cisme et lien social. Enquête autour de Musonius Rufus, Paris, 2014 (Les anciens et les modernes, 14).

Morel, P.-M. 2000 : « Épicure, l'histoire et le droit », Revue des études anciennes, 102/3-4 (2000), p. 393-411.

DOI : https://doi.org/10.3406/rea.2000.4802

Morel, P.-M. 2007 : « Les communautés humaines », dans A. Gigandet, P.-M. Morel (éd.), Lire Épicure et les épicuriens, Paris, 2007 (Quadrige), p. 167-186.

Morel, P.-M. 2011 (éd.) : Épicure, Lettres, maximes et autres textes, Introduction, traduction, dossier et notes, Paris, 2011 (GF, 1479).

Morel, P.-M. 2017 : « La Terre entière, une seule patrie : Diogène d'CEnoanda et la politique », dans J. Hammerstaedt, P.-M. Morel, R. Güremen (éd.), Diogenes of Oinoanda = Diogène d'CEnoanda. Epicureanism and Philosophical Debates = Épicurisme et controverses, Leuven, 2017 (Ancient and Medieval Philosophy. Series 1, 55), p. 221-240.

Nussbaum, M. 1994 : The Therapy of Desire: Theory and Practice in Hellenistic Ethics, Princeton, 1994.

Procopé, J. F. 1998 : « Epicureans on Anger », dans J. Sihvola, T. EngbergPedersen (éd.), The Emotions in Hellenistic Philosophy, Dordrecht/Boston/ London, 1998 (The New Synthese Historical Library, 46), p. 171-196.

Prost, F. 2012 : «La mesure des affections dans l'épicurisme », PALLAS, 88 (2012), p. 71-81.

DOI : $10.4000 /$ pallas. 2463

Purinton, J. 1993 : « Epicurus on the telos », Phronesis, 38/3 (1993), p. 281-320. DOI : https://doi.org/10.1163/156852893321052316

Roskam, G. 2007 : "Live unnoticed" = $\Lambda \alpha \dot{\theta} \theta \beta 1 \omega \sigma \alpha \varsigma$. On the Vicissitudes of an Epicurean Doctrine, Leiden/Boston, 2007 (Philosophia antiqua, 111).

SALEM, J. 1990 : La Mortn'est rien pour nous. Lucrèce et l'éthique, Paris, 1990 (Bibliothèque d'histoire de la philosophie. Nouvelle série).

Schiesaro, A. 2007 : « Lucretius and Roman Politics and History », dans 
S. Gillespie, Ph. Hardie (éd.), The Cambridge Companion to Lucretius, Cambridge, 2007 (Cambridge Companions to Literature), p. 41-58.

Schofield, M. 1991 : The Stoic Idea of the City, Chicago-London, 1991 (2 ${ }^{\text {nde }}$ éd. 1999).

Schrijvers, P. H. 1999 : Lucrèce et les sciences de la vie, Leiden-Boston-Köln, 1999 (Mnemosyne. Supplementum, 186).

Usener, H. 1887 (éd.) : Epicurea, Leipzig, 1887.

Veillard, C. 2016 : « Hiéroclès, les devoirs envers la patrie et les parents », dans J.-B. Gourinat (éd.), L'éthique du stoïcien Hiéroclès, Villeneuve d'Ascq, 2016 (Philosophie antique. Hors-Série, 1), p. 105-143.

Verde, F. 2010 (éd.) : Epicuro, Epistola a Erodoto, intr. di E. Spinelli, trad. e commento di F. V., Roma, 2010 (Classici, 5).

VERDE, F. à paraître : « Il saggio epicureo e il controllo delle passioni », dans Chr. Veillard, O. Renaut et D. El Murr (dir.), Les philosophes face au vice, de Socrate à Augustin, Leiden (Philosophia Antiqua) à paraître.

WACHT, M. 1991 : Concordantia in Lucretium, Hildesheim-Zurich-New York, 1991 (Alpha-Omega. Reihe A, Lexika, Indizes, Konkordanzen zur klassischen Philologie, 122). 